\title{
The Latest Innovation on FE-SEM and its Applications
}

\author{
Shuichi Takeuchi ${ }^{1}$, Hirofumi Sato ${ }^{1}$, Osamu Takagi ${ }^{1}$, David Hoyle ${ }^{2}$ \\ ${ }^{1 .}$ Hitachi High-Technologies Corp., 882, Ichige, Hitachinaka, Ibaraki 312-8504, Japan \\ 2. Hitachi High-Technologies Canada, inc., 89 Galaxy Blvd, Suite 14 Toronto, Ontario M9W 6A4, \\ Canada
}

Nano materials to be used for batteries and semiconductor devices are being made as composite materials in order to improve its functionality and durability. The latest FE-SEM has been developed to meet these demands by continuous improvements not only on fundamental technologies but also addressing imaging capabilities.

In order to obtain optimized contrast on potential contrast, compositional contrast at high magnification for the above demands, 1) Spreading energy width with emitter, 2) Energy separation of a signal would be key terms for the innovators.

As a field proven technology of Hitachi Cold Field Emission Gun maintains high brightness and high stability with 0.2 to $0.3 \mathrm{eV}$ energy spreading while implementing newly innovative evacuation system, the latest FE-SEM would achieve further advanced performance to maintain reliable image observation and analysis for all scientific segments.

And newly developed energy filtering function can control charge-up phenomena and improve detection capabilities on SE/BSE signals. For example, the potential contrast from SE signal is very useful for observation on advanced materials such as battery electrode and effective information from compositional contrast would also be available from BSE signal which would be of use for material differentiation of semiconductor devices. The filter function would be effectively utilized to select any signals for observation and specimen selection. Overall imaging capabilities such as topography, composite, crystal, and potential contrast would be utilized to obtain any useful information for advanced material research.

In this study the latest Hitachi FE-SEM and EDS system are utilized for the evaluations. Figure 1 shows the observation and analysis on anode electrode of rechargeable lithium-ion batteries, and physical characteristics from potential differences on specimen surface at the ultra low landing voltage of $300 \mathrm{~V}$ are available from the information both bright contrast of carbon material (a) and dark contrast of resin component (b)[1]. EDS mapping results are shown as (c) $\mathrm{C}-\mathrm{K} \alpha$, (d) O-K $\alpha$, (e) F-K $\alpha$, and (f) Ni-L $\alpha$ at accelerating voltage of $2,000 \mathrm{~V}$. Low accelerating voltage analysis enables us to analyze less beam damage even on resin specimens and high spatial resolution on fine surface structure. Therefore the latest innovation on FE-SEM and EDS system provides multiple evaluation results on rechargeable lithium-ion batteries.

[1] S Takeuchi et al, Microsc. Microanal. Vol.16(Suppl S2) (2010) p.p.616-617. 

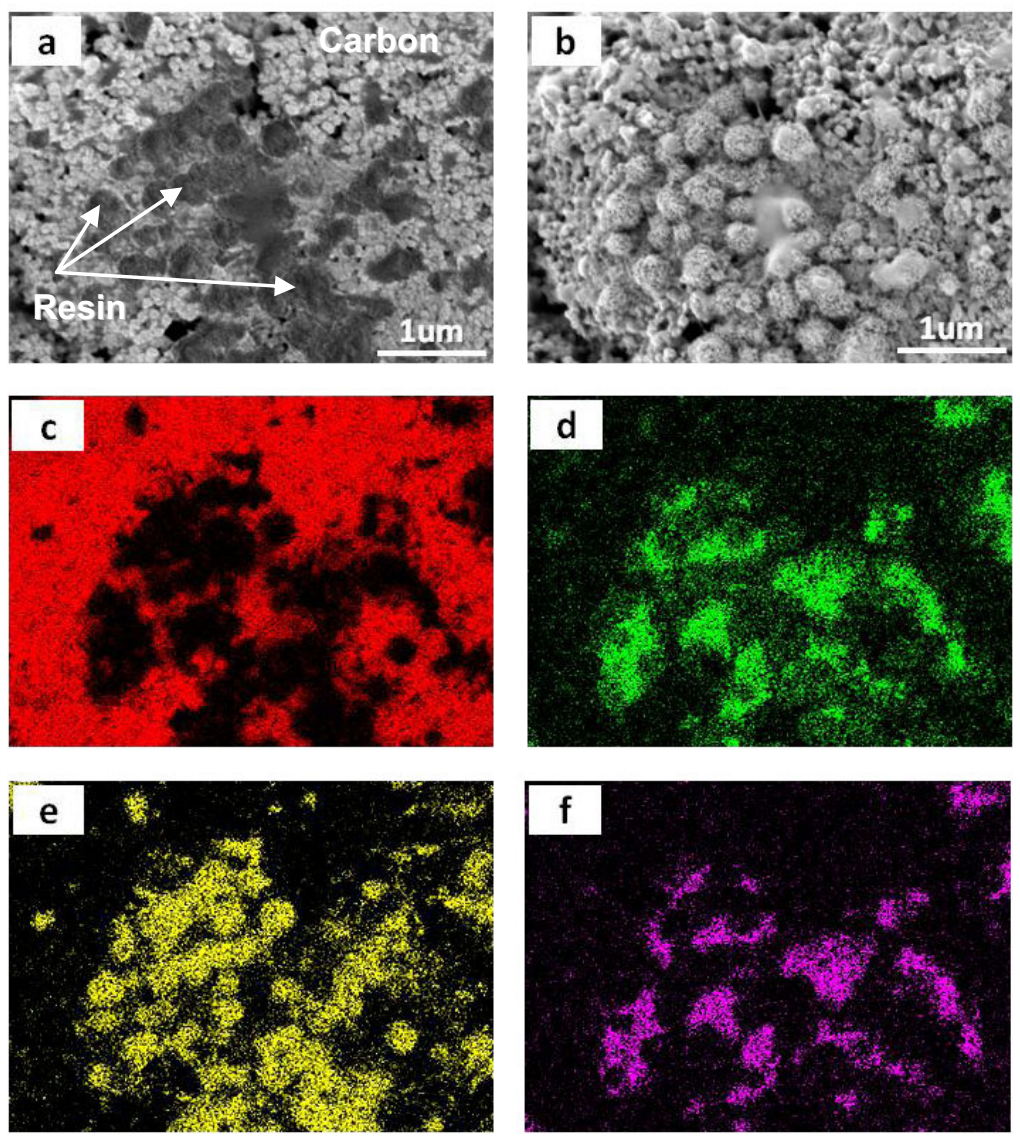

Figure 1. Evaluation results of anode electrode for rechargeable lithium-ion batteries at same field of view.

Potential contrast (a) and topographic contrast (b) imaging under the ultra low landing of voltage $300 \mathrm{~V}$. Bruker XFlash Flat QUAD 5060F EDS mapping results (c) C-K $\alpha$, (d) O-Ka, (e) F-K $\alpha$ and (f) Ni-L $\alpha$ at accelerating voltage $2 \mathrm{kV}$. 